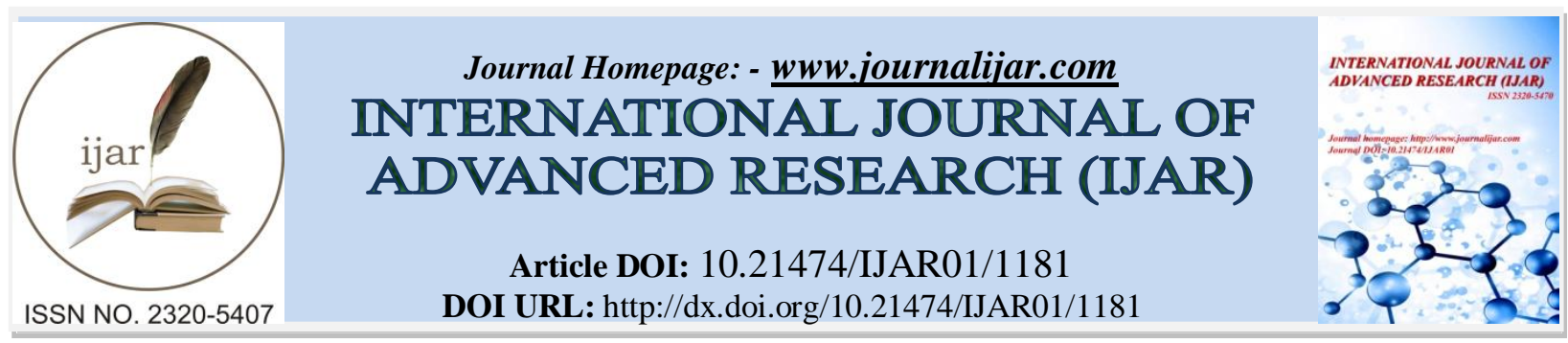

RESEARCH ARTICLE

\title{
SEROLOGICAL SECREENING OF DIABETES MELLITUS FORCELIAC DISEASE IN PEDIATRICS.
}

Mohammad Abud Darweesh.

Pediatric Consultant, Al-Sadder Teaching Hospital, Department of Pediatrics, College of Medicine, Maysan University, Maysan, Iraq.

\section{Manuscript Info}

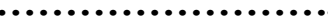

Manuscript History

Received: 12 June 2016

Final Accepted: 19 July 2016

Published: August 2016

Key words:-

Celiac disease

Diabetic mellitus

Serology tests

Transeglutaminase

\section{Abstract}

Objective. This study conduct to determine the celiac disease prevalence in diabetic mellitus cases, among sex, age group, duration of diagnosis and family history.

Method. Forty five patients who had presented to the diabetic center in Al-Sadder Teaching Hospital and privet pediatric clinic between August 2014 to April 2015. The study group comprised of 21 males and 24 females with age range from 9 months to 16 years. Whom had the T1DM. Serological tests done by using the Tissue transeglutaminase tTG-A.

Findings. The positive results of celiac disease in patient with T1DM was $17(37.78 \%)$ cases, The male to female ratio of celiac disease was $1: 1$. According to the age groups, the most age group was $9-12$ years as $7(15.56 \%)$ cases.

Conclusion. The increase in the malignancy incidence was statistically significant in cases in which the dominant nodule diameter was $>4 \mathrm{~cm}$. However, the frequency of thyroid papillary carcinoma was determined as

$12.9 \%$ in MNG patients in whom the DND was $>4 \mathrm{~cm}$.

Copy Right, IJAR, 2016,. All rights reserved.

\section{Introduction:-}

Diabetes mellitus is a common, chronic, metabolic syndrome characterized by hyperglycemia as a cardinal biochemical feature. Type 1 diabetes mellitus (T1DM) is the most common endocrine metabolic disorder of childhood and adolescence, with important consequences for physical and emotional development. Individuals with T1DM confront serious lifestyle alterations that include an absolute daily requirement for exogenous insulin, the need to monitor their own glucose level, and the need to pay attention to dietary intake. Morbidity and mortality stem from acute metabolic derangements and from long-term complications (usually in adulthood) that affect small and large vessels resulting in retinopathy, nephropathy, neuropathy, ischemic heart disease, and arterial obstruction with gangrene of the extremities. The acute clinical manifestations are due to hypo-insulinemic hyperglycemic ketoacidosis [1]. T1DM is associated with other autoimmune diseases such as thyroiditis, celiac disease, multiple sclerosis, and Addison disease. Autoimmune diseases associated with type 1 diabetes should be sought, including celiac disease (by tissue transglutaminase $\operatorname{IgA}$ and total $\operatorname{IgA}$ ) and thyroiditis (by anti-thyroid peroxidase and antithyroglobulin antibodies) [2]. T1DM accounts for about 10\% of all diabetes, affecting about 15 million in the world. 
The incidence of T1DM is highly variable among different ethnic groups. The overall age-adjusted incidence of type 1 DM varies from 0.7/100,000 per year in Karachi (Pakistan) to about 40/100,000 per year in Finland. Peaks of presentation occur in 2 age groups: at 5-7 year of age and at the time of puberty. A growing number of cases are presenting between 1 and 2 year of age. The 1st peak may correspond to the time of increased exposure to infectious agents coincident with the beginning of school; the 2nd peak may correspond to the pubertal growth spurt induced by gonadal steroids and the increased pubertal growth hormone secretion (which antagonizes insulin) [1]. The etiology of T1DM is multifaceted and may be divided into genetic and environmental etiology and possible gene environment interactions. Genetic susceptibility increases predisposition for triggering islet autoimmune responses. Although more than $85 \%$ of T1DM occurs in individuals with no previous first-degree family history, the risk among first- degree relatives is about 15 times higher than the general population. An affected father confers a $6-9$ $\%$ risk of T1DM to his offspring compared to $2-4 \%$ if the mother is affected and up to $30 \%$ risk if both parents are affected. The most prominent environmental factors include maternal factors, viral infections, dietary, high birth weight and growth rate, psychological stress and toxic substances [3]. Coeliac disease is an immunologically mediated inflammatory disorder of the small bowel occurring in genetically susceptible individuals [4]. CD is an autoimmune disorder that stimulates $\mathrm{T}$ cells -white blood cells essential for healthy immunity-to inflame the mucosa or lining of the small bowel and destroy the villi in the small intestine, preventing the absorption of nutrients from food into the blood stream. We are not born with $\mathrm{CD}$, but it occurs in people who are genetically predisposed and can be triggered by a viral or bacterial infection, pregnancy,or the consumption of the protein gluten present in the grains of wheat, barley, and rye. The age of onset varies from infancy to the elderly, and the symptoms can be very subtle or obvious [5]. The prevalence of celiac disease is globally 1\%, but large variations among countries have been shown [6]. Celiac disease can manifest with the classic constellation of symptoms and signs of a malabsorption syndrome [7]. An infant may show symptoms when he or she is introduced to grains at an early age. The symptoms may include diarrhea, constipation, foul-smelling stools, fatigue, slow growth pattern, irritability, and even a swollen belly from malnutrition. An older adult frequently presents with anemia from no apparent cause [5]. Anti-tissue transglutamiase antibodies are highly sensitive and specific for diagnosis of celiac disease [8]. The female-to-male ratio is $2: 1$ [9]. The prevalence of biopsy- confirmed celiac disease (CD) in patients with T1DM ranges from $3 \%$ to $7 \%$, compared to $1 \%$ in the general population. Many children with diabetes are asymptomatic but are positive for specific serologic markers of CD such as autoantibodies to tissue transglutaminase. Most of the children with diabetes and CD still remain undiagnosed, despite intestinal symptoms and/or short stature in about half of the cases. Clinical manifestations of $\mathrm{CD}$ are diverse, vary with age and may overlap with functional disorders. Some of the manifestations, such as delayed growth and puberty, decreased bone mineralization, abdominal pain and abnormal liver function tests, may overlap with those of poorly controlled diabetes. Therefore, physicians and other health care providers need to consider $\mathrm{CD}$ in the differential diagnosis and many have argued for a routine transglutaminase screening in children with T1DM. T1DM and CD share HLA and non- H LA susceptibility genes. The prevalence of transglutaminase autoimmunity is highest in those with the HLA- DR3, DQB1* 0201 haplotype. One in four children with diabetes homozygous for this haplotype and 12\% of the heterozygotes are positive for transglutaminase autoantibodies. Most patients with T1DM with CD are transglutaminase autoantibody - positive at the initial screening, although new cases develop during follow-up. All patients should be screened for immunoglobulin A ( $\operatorname{IgA}$ ) transglutaminase-autoantibodies at onset of diabetes and, if negative and asymptomatic, rescreened every other year. If transglutaminase autoantibodies are strongly and persistently positive, biopsy is recommended even in a completely asymptomatic patient. By contrast, patients with low to moderately positive transglutaminase autoantibody levels may have false - negative biopsy and may be falsely reassured that they do not have CD and forego further follow-up. Untreated CD may pose problems with diabetes management, including increased risk of hypoglycemia and chronic diarrhea which is difficult to differentiate from that caused by autonomic neuropathy in adult patients [3]. Because of this strong association it is recommended that patients with type 1 diabetes should be screened for concomitant celiac disease [9].

\section{Patients and Methods:-}

A descriptive study was conducted in diabetic center in Maysan province, Al-Sadder Teaching Hospital and from my supervisor clinic referrals. The data was collected from the patients in all of these places by my efforts. The study was performed by percentage calculation and frequency.

\section{Study Population:-}

A total number of 45 individuals who having T1DM encountered in the study, during a period from the first of August 2014 to the first of April 2015. The study group comprised of 21 males and 24 females with age range from 9 months to 16 years. 


\section{Collection of Samples:-}

The blood samples collected from the patients under aseptic technique, and then immediately transported to the serological laboratory in AL-Sadder Teaching Hospital for further processing. The name, age and sex were clearly mentioned on the container containing specimen.

\section{Principle of the test:-}

Tissue transeglutaminase tTG-A is a solid phase enzyme immunoassay for the quantitative and qualitative detection of antibodies against neo-epitopes of tissue transglutaminase (tTG) in human serum. The assay employing human recombinant transglutaminase cross linked with gliadin-specific peptides displays neo-epitopes of tTG which ensures a significantly increased sensitivity and specificity of the test. The assay is a tool for the diagnosis and monitoring of celiac disease(gluten-sensitive enteropathy). Serum samples diluted 1:101 are incubated in the microplates coated with the specific antigen. Patient's antibodies, if present in the specimen, bind to the antigen. The unbound fraction is washed off in the following step. Afterwards anti-human immunoglobulins conjugated to horseradish peroxidase (conjugate) are incubated and react with the antigen-antibody complex of the samples in the microplates. Unbound conjugate is washed off in the following step. Addition of TMB-substrate generates an enzymatic colorimetric (blue) reaction, which is stopped by diluted acid (color change to yellow). The rate of color formation from chromogen is a function of the amount of conjugate bound to the antigen-antibody complex and this is proportional to the initial concentration of the respective antibodies in the patient sample.

\section{Procedure:-}

Preparation prior to pipetting. Dilute concentrated reagents, then dilute the concentrated sample buffer 1:5 with distilled water. Dilute the concentrated wash buffer 1:50 with distilled water. Then dilute serum samples with sample buffer and mix well. The washing start by prepare $20 \mathrm{ml}$ of diluted wash buffer per 8 wells. The automated washing, consider excess volumes required or setting up the instrument and dead volume of robot pipette. The manual washing, doing by discard liquid from wells by inverting the plate. knock the micro-well frame with wells downside vigorously on clean adsorbent paper. Pipette 300 micro ml. of diluted wash buffer, wait for 20 seconds. Repeat the whole procedure twice again. In micro-plates, by calculate the number of wells required for the test. Remove unused wells from the frame, replace and store in the provided plastic bag, together with desiccant, seal tightly $\left(2-8^{\circ} \mathrm{C}\right)$.

\section{Work flow:-}

Pipette $100 \mu \mathrm{ml}$ of each patient's diluted serum into designated micro-wells. Then pipette $100 \mu \mathrm{ml}$ calibrators OR cut-off calibrator, negative and positive controls into the designated wells, then incubate for 30 minutes at 20-32 C. Wash $3 \mathrm{x}$ with $300 \mu \mathrm{ml}$ washing buffer (diluted 1:50). Pipette other $100 \mu \mathrm{ml}$ conjugate into each well, then incubate for 30 minutes at 20-32C. Wash 3x with $300 \mu \mathrm{ml}$ washing buffer (diluted 1:50). Pipette 100 micro mil TMB substrate into each well and incubate for 30 minutes at 20-32 C, protected from intense light. Then pipette $100 \mu \mathrm{ml}$ stop solution into each well, using the same order as pipetting the substrate. Incubate 5 minutes minimum. Agitate plate carefully for $5 \mathrm{sec}$ and read absorbance at $450 \mathrm{~nm}$ within 30 minutes [10].

\section{Result and Discussion:-}

The positive results of celiac disease using serological test in patient with T1DM was 17 from the total cases 45 as $37.78 \%$ percent as showed in table 1 .

Table 1. Number of positive cases of celiac disease in patients with diabetes mellitus

\begin{tabular}{|l|l|l|}
\hline Serological test & Number of the cases of celiac disease & Percentage \\
\hline Positive & 17 & 37.78 \\
\hline Negative & 28 & 62.22 \\
\hline Total & 45 & 100 \\
\hline P $>0.01$ & \multicolumn{2}{l|}{} \\
\hline
\end{tabular}

The results of celiac disease by serology in patients of T1DM According to the gender, showed in table 2. 
Table 2. The distribution of celiac disease according to the gender of patients.

\begin{tabular}{|l|l|l|l|l|l|l|}
\hline \multirow{2}{*}{ Gender } & Test results & Total & $\%$ \\
\cline { 2 - 7 } & Positive & $\%$ & Negative & $\%$ & & \\
\hline Male & 9 & 20.00 & 12 & 26.67 & 21 & 46.67 \\
\hline Female & 8 & 17.78 & 16 & 35.55 & 24 & 53.33 \\
\hline Total & 17 & 37.78 & 28 & 62.22 & 45 & 100 \\
\hline P $>0.5$ & \multicolumn{7}{l|}{} \\
\hline
\end{tabular}

The celiac disease distribution according to the age groups, the percent of age group < 4 years was $4.44 \%$, and the age 4-8 years the percent was $11.11 \%, 9-12$ years was $15.56 \%$, and the percent of age group 13-16 years was 6.67 $\%$, in patients with T1DM, P value of no significance as showed in table 3.

Table 3. The distribution of celiac disease according to the age group

\begin{tabular}{|c|c|c|c|c|c|c|}
\hline \multirow{3}{*}{ Age } & \multicolumn{4}{|c|}{ Test results } & \multirow{3}{*}{ Total } & \multirow[t]{3}{*}{$\%$} \\
\hline & \multicolumn{2}{|l|}{ Positive } & \multicolumn{2}{|c|}{ Negative } & & \\
\hline & Number & $\%$ & Number & $\%$ & & \\
\hline$<4$ years & 2 & 4.44 & 2 & 4.44 & 4 & 8.89 \\
\hline 4-8 years & 5 & 11.11 & 10 & 22.22 & 15 & 33.33 \\
\hline $9-12$ years & 7 & 15.56 & 6 & 13.34 & 13 & 28.89 \\
\hline $13-16$ years & 3 & 6.67 & 10 & 22.22 & 13 & 28.89 \\
\hline Total & 17 & 37.78 & 28 & 62.22 & 45 & 100 \\
\hline
\end{tabular}

Among patients with T1DM, celiac disease according to the duration of diagnosis of DM, the percent of positive cases in patients with duration of $<1$ year was $6.67 \%$, while the percent of positive cases in patients with duration $>$ 1 year was $31.11 \%$.

Table 4. The distribution of celiac disease according to the duration of diagnosis of T1DM.

\begin{tabular}{|c|c|c|c|c|c|c|}
\hline \multirow[t]{2}{*}{ Duration of diagnosis } & \multicolumn{6}{|l|}{ Test } \\
\hline & Number & $\%$ & Number & $\%$ & Total & $\%$ \\
\hline$<1$ year & 3 & 6.67 & 11 & 24.44 & 14 & 31.11 \\
\hline$>1$ year & 14 & 31.11 & 17 & 37.78 & 31 & 68.89 \\
\hline Total & 17 & 37.78 & 28 & 62.22 & 45 & 100 \\
\hline
\end{tabular}

The total cases with family history of DM were 25 , twelve of them were positive for celiac disease using serological test, and 13 of them were negative for celiac disease.

Table 5. The distribution of celiac disease according to family history of DM.

\begin{tabular}{|l|l|l|l|l|l|l|}
\hline \multirow{2}{*}{$\begin{array}{l}\text { Test results of } \\
\text { CD }\end{array}$} & Family history of DM & Total & $\%$ \\
\cline { 2 - 5 } & Positive & $\%$ & Negative & $\%$ & & \\
\hline Positive & 12 & 26.67 & 5 & 11.11 & 17 & 37.78 \\
\hline Negative & 13 & 28.89 & 15 & 33.33 & 28 & 62.22 \\
\hline Total & 25 & 55.56 & 20 & 44.44 & 45 & 100 \\
\hline P $<0.05$ & \multicolumn{7}{|l|}{} \\
\hline
\end{tabular}

The total cases with family history of celiac disease were 7 , five cases were positive for celiac disease using serological test, two of them were negative, the $\mathrm{P}$ value of significance. 
Table 6. The distribution of celiac disease according to the family history of celiac disease.

\begin{tabular}{|l|l|l|l|l|l|l|}
\hline \multirow{2}{*}{ Test results of CD } & Family history of & Total & $\%$ \\
\cline { 2 - 7 } & Positive & $\%$ & Negative & $\%$ & & \\
\hline Positive & 5 & 11.11 & 12 & 26.67 & 17 & 37.78 \\
\hline Negative & 2 & 4.44 & 26 & 57.78 & 28 & 62.22 \\
\hline Total & 7 & 15.56 & 38 & 84.44 & 45 & 100 \\
\hline P> 0.045 & \multicolumn{5}{|l}{} \\
\hline
\end{tabular}

Diabetes mellitus is a common metabolic disorder. Celiac disease is an autoimmune disease associated with other autoimmune diseases. There is association between diabetes mellitus type I and celiac disease, about $37.78 \%$ of patients with diabetes mellitus type I have celiac disease by using of serological test, while one in four children with diabetes homozygous for this haplotype and $12 \%$ of the heterozygotes are positive for transglutaminase autoantibodies. Most patients with celiac disease have the silent form. Celiac disease is more predominance in males than females, while The female-to-male ratio is 2:1. Celiac disease prevalence more in age group (9-12) years. Celiac disease more likely to occur in T1DM patients with family history of diabetes mellitus. Because of celiac disease is genetically predisposed, there is a strong association between celiac patients whose already have T1DM with family history of celiac disease.

\section{Recommendations:-}

Patients with diabetes mellitus type I must be aware about the control of blood sugar, regular measuring of glucose in the blood, also compliance of the treatment and the right way for injection of insulin. The physician should be aware about the high risk of celiac disease in patients with type $1 \mathrm{DM}$, even in the absence of symptoms and signs of celiac disease. Patients who have celiac disease should be restricted from taking gluten (wheat, rye and barley) for life. Educate the parents about the association of T1DM with other autoimmune disease such as celiac disease, so patients with diabetes mellitus type I should be screened annually for celiac disease. For consolidation of diagnosis of celiac disease ,so patients with positive serological test should be send for biopsy. It should be keep in mind, the long term risk of celiac disease is lymphoma and intestinal malignancies.

\section{References:-}

1. Alemzadeh R and Wyatt DT. Diabetes mellitus. In: Kliegman RM, ed.Nelson Textbook of Pediatrics. 18th edition. Saunders; 2007:chap 590.

2. Nicholas J, (2014). Diabetes Mellitus, Section XXIII, Nelson Essentials Of Pediatrics 5th edition.

3. David A. W, Timothy M. C, John D. F, Edward J., and M.D. Benz, (2003). Oxford Textbook of Medicine, 4th edition.

4. Palmer K R and I D Penman, (2008). Alimentary Tract And Pancreatic Disease, Chapter 22, Davidson's Principles \& Practice Of Medicine, 21st edition.

5. Sylvia L L, Bower, R N, Marry K S and Steve P, (2009). What Is Celiac Disease, Chapter 1, Celiac Disease A guide To Living With Gluten Intolerance, Second edition.

6. Lionetti E, Catassi C. New clues in celiac disease epidemiology, pathogenesis, clinical manifestations and treatment, Int Rev, Immunology. 2011; 30: 219.

7. Ebert E C. Mal digestion and mal absorption, Dis Mon. Chapter VI, Gastrointestinal disease, Goldman :Cecil Essential Of Medicine, 6th Edition. 2001; 47 :49.

8. Dietrich W , Ehnis T , Bauer M. Identification of tissue transglutaminase as the auto antigen of celiac disease. Nat Med. 1997.

9. Epidemiology, WGO Practice Guidelines, Celiac Disease, World Gastroenterology Organization, 2007.

10. Aeskulisa, The Diagnostic Tool that Works, Instruction Manual. 